\title{
Assessment of Minerals in Phoenix Dactylifera L. as Determined by Inductively Coupled Plasma Optical Emission Spectrometry using ANOVA and PCA
}

\author{
Khalilah Badarusham, Nurul Elma Sabri, Shamala Salvamani, \\ Mohd Sukri Hassan, Zaiton Hassan, Roshada Hashim
}

\begin{abstract}
Dates (Phoenix dactylifera L.) is one of mankind's oldest cultivated plants. It is known to be the most important fruit tree particularly in the Middle Eastern and Asian countries due to its natural sources of nutrition. The element composition of 10 commercially available dates and its pit samples purchased from local market were determined and evaluated using multivariate analysis technique. Samples were digested using nitric acid and hydrochloric acid and analyzed using Inductively Coupled Plasma-Optical Emission Spectrometry (ICP-OES). Potassium, magnesium, calcium and sodium were found to be the major elements in both flesh and pit samples. Comparison between minor elements in both samples showed that the flesh samples contained higher levels of aluminium, barium and selenium while other minor elements were higher in the pit samples. Principal Components Analysis (PCA) and Analysis of Variance (ANOVA) were performed on multivariate dataset of the flesh and the pit samples separately to observe the relationship between samples.

Index Terms: ANOVA, date fruit, inductively coupled plasma optical emission spectroscopy (ICP-OES), mineral elements, principal components analysis (PCA).
\end{abstract}

\section{INTRODUCTION}

Phoenix dactyliferaL. or commonly known as the date palm is a tropical and subtropical tree that belongs to Palmae (Arecaceae) family and is one of the earliest plants cultivated by humans [1], [2]. Date palms are also called Sugar Palm (English), Nakhal (Arabic), Hurmaağact (Turkish), khrma(Persian), Khajur (Hindi and Urdu), Karchuram (Tamil, Malayalam) and Karjura (Kannada). Dates are reported to contain essential minerals such as sodium $(\mathrm{Na})$, potassium $(\mathrm{K})$, phosphorus $(\mathrm{P})$, calcium $(\mathrm{Ca})$, magnesium $(\mathrm{Mg})$ and vitamins, for example, vitamin $\mathrm{C}$ and

Revised Manuscript Received on July 10, 2019.

Khalilah Badarusham, Faculty of Science and Technology, Universiti Sains Islam Malaysia, 71800 Bandar Baru Nilai, Negeri Sembilan, Malaysia.

Nurul Elma Sabri, Faculty of Science and Technology, Universiti Sains Islam Malaysia, 71800, Bandar Baru Nilai, Negeri Sembilan, Malaysia.

Shamala Salvamani, Faculty of Science and Technology, Universit Sains Islam Malaysia, 71800 Bandar Baru Nilai, Negeri Sembilan, Malaysia.

Mohd Shukri Hassan, Faculty of Science and Technology, Universiti Sains Islam Malaysia, 71800 Bandar Baru Nilai, Negeri Sembilan, Malaysia.

Zaiton Hassan, Faculty of Science and Technology, Universiti Sains Islam Malaysia, 71800 Bandar Baru Nilai, Negeri Sembilan, Malaysia.

Roshada Hashim, Faculty of Science and Technology, Universiti Sains Islam Malaysia, 71800 Bandar Baru Nilai, Negeri Sembilan, Malaysia. vitamin E [3], [4]. Some of the minerals are essential in bone metabolism and enzymatic activity [5]. Minerals in food can be grouped into macro (major) or micro (minor) minerals, the macro-minerals include calcium (Ca) phosphorus $(\mathrm{P})$, potassium $(\mathrm{K})$, magnesium $(\mathrm{Mg})$, sodium (Na) and chlorine $(\mathrm{Cl})$, while the micro-minerals include iron $(\mathrm{Fe})$, copper $(\mathrm{Cu})$, cobalt $(\mathrm{Co})$, iodine $(\mathrm{I})$, zinc $(\mathrm{Zn})$, manganese $(\mathrm{Mn})$, molybdenum (Mo), fluoride $(\mathrm{F})$, chromium (Cr), selenium (Se), nickel (Ni) and sulfur (S) [6]. The macro-minerals are required in amounts greater than $100 \mathrm{mg} / \mathrm{dl}$ and the micro-minerals are required in amounts less than $100 \mathrm{mg} / \mathrm{dl}$ [7]. $\mathrm{Mg}, \mathrm{Cu}, \mathrm{Se}, \mathrm{Zn}, \mathrm{Fe}, \mathrm{Mn}$ and Mo are important co-factors found in the structure of certain enzymes and are indispensable in numerous biochemical pathways. $\mathrm{Na}, \mathrm{K}$ and $\mathrm{Cl}$ play a crucial role in the maintenance of osmotic balance between cells and the interstitial fluid [5], [8]. The minor elements like $\mathrm{Cu}, \mathrm{Fe}, \mathrm{Se}$ and $\mathrm{Zn}$ have shown immunity in HIV-1 patients by lowering the rate of progression to AIDS. The quantity of micronutrients intake is also important for energy and protein sources [9].

The macro and micro minerals contents in date flesh have been reported previously by [10]-[12] with different varieties of dates samples and the instrument used. Inductively coupled plasma is one of the analytical technique that is more frequently reported [13]-[16] to analyze metals in the plant materials and food samples. In this study, Inductively Coupled Plasma-Optical Emission Spectroscopy (ICP-OES) was used instead of atomic absorption spectroscopy (AAS) technique that is used previously by several researchers in determining metals concentration. ICP-OES has a very good detection limit for most elements, small sample usage and many samples can be run at a time compared to AAS. In addition, ICP-OES has characteristics such as high reliability, high sensitivity and relatively less affected by chemical interference [17], [18]. In [19] measured the concentrations of $\mathrm{K}, \mathrm{Fe}$ and $\mathrm{Mg}$ in date fruit using ICP-OES, while in [20], [21] employed 


\section{ASSESSMENT OF MINERALS IN PHOENIX DACTYLIFERA L. AS DETERMINED BY INDUCTIVELY COUPLED PLASMA OPTICAL EMISSION SPECTROMETRY USING ANOVA AND PCA}

ICP-OES to determine the mineral profile of date fruits and seeds, respectively in Saudi Arabia. Different techniques and methods were used in mineral determination with different dates samples, however, only a few varieties have been investigated so far and very little information has been published particularly on dates which are available in Malaysia markets.

In elemental analysis, the results obtained are in multivariate dataset which are huge and complex. Previous works on date samples used basic statistic to analyze their data, whereas in this study, one way-Analysis of Variance (ANOVA) and Principal Components Analysis (PCA) were used to evaluate the obtained data. PCA is a multivariate data analysis technique for evaluation and characterization of complex analytical data [22]. In [23] applied PCA to evaluate the minerals content of 50 Chinese medicinal herbs after acid digestion using flame AAS. In [24] determined the differences between the minerals of Zhongning wolfberry among the regions using PCA and Linear Discriminant Analysis (LDA). Furthermore, PCA combined with cluster analysis are useful tools to classify mineral content as shown by [25] for fruit juices and nectars according to fruit type and the geographical origin; and in [26] for minerals and heavy metals contents in raw honeys from four different bee species to distinguish the honeys by its species using AAS and inductively coupled plasma-mass spectrometry (ICPMS), respectively. PCA has also been successfully used in differentiating different grape cultivars [27] and the peel, pulp and seed of Passifloraedulis $f$. flavicarpaand Passifloracincinnata cultivated in Bahia state, Brazil based on their mineral contents [28].

In Malaysia, dates are more desired fruit during the Ramadan month when Muslim performing their fast. The fruit also consumed by many people regardless of race and religion. Prices differ by the types and grades of dates. Suppliers in Malaysia usually import dates in bulk, repack into small packaging and market them under different names and prices. Unfortunately, the contents of only several elements such as $\mathrm{K}, \mathrm{Ca}, \mathrm{Mg}$ and $\mathrm{Fe}$ are labeled while information of other major and minor elements in dates is not included. Hence, this study was conducted to measure the elements composition in a variety of dates (and their pits) available in Malaysia markets using ICP-OES. ANOVA and PCA analyses provide information that will reveal the differences between the varieties of dates and thus allow a more informed decision among Malaysian consumers in their selection of dates based on the quality. The dates pits were also included in this study since they are used as mineral sources in animal feeds or turned into non caffeinated coffee.

\section{MATERIALS AND METHODS}

\section{Chemical Reagents and Solutions}

All chemical reagents were of analytical grade. Ultrapure water (18.2 M $\mathrm{M} . \mathrm{cm}^{-1}$ ) from a Milli-Q system (Millipore, MA, USA) was used to prepare all the solutions. Stock solutions of the elements; Aluminium (Al), Barium (Ba), Calcium (Ca), Copper $(\mathrm{Cu})$, Iron $(\mathrm{Fe})$, Potassium $(\mathrm{K})$, Magnesium (Mg), Manganese (Mn), Sodium(Na), Nickel $(\mathrm{Ni})$, Lead (Pb), Zinc (Zn), Selenium (Se) (1000 mg.L ${ }^{-1}$,

Merck, Darmstadt, Germany) were used to prepare working standard solutions by dilution with $1 \%(\mathrm{v} / \mathrm{v})$ nitric acid. All laboratories glassware used were immersed in $10 \% \mathrm{v} / \mathrm{v}$ of nitric acid solution for $12 \mathrm{hr}$ for decontamination prior to use and then rinsed several times with deionized water. Nitric acid (65\% v/v, Merck, Darmstadt, Germany) and hydrochloric acid $(65 \% \mathrm{v} / \mathrm{v})$, were used for acid digestion.

\section{Instrumentation}

ICP-OES model Optima 4300DV (PerkinElmer Inc., USA) was used with axial viewing for determination of elemental composition. The ICP-OES was set-up with the following conditions:

$\begin{array}{ll}\text { RF Power } & : 1300 \mathrm{Watt}^{-1} \\ \text { Plasma gas rate (Ar) } & : 8.0 \mathrm{Lmin}^{-1} \\ \text { Auxiliary gas rate } & : 0.2 \mathrm{~L} \mathrm{~min}^{-1} \\ \text { Nebulizer gas rate } & : 0.55 \mathrm{~L} \mathrm{~min}^{-1} \\ \text { Pump flow rate } & : 1.5 \mathrm{~mL} \mathrm{~min}^{-1}\end{array}$

\section{Sample Preparation}

Ten samples of imported date fruits (Ajwa, Medjool, Mariami, Sunsweet, Nourina, Mabroom, Khudry, Sunseed, Degletnour and Mesir) were purchased from a local supermarket. The samples were stored in refrigerated bags to prevent the proliferation of fungi and bacteria. Samples were washed several times with deionized water to remove any impurities and then oven dried at $50^{\circ} \mathrm{C}$ for 1 hour. All the samples were blended using a, 2 speed, $230 \mathrm{~V} / 50 \mathrm{~Hz}, 1 \mathrm{~L}$ of Waring blender (National, Japan) to homogenize the samples.

\section{Ash and Elements Determination}

The samples (approximately $2.0 \mathrm{~g}$ ) were dried in the oven (Binder, FisherScientific, Malaysia) at $115^{\circ} \mathrm{C}$ for 1 hour. They were placed in a muffle furnace (Carbolite, England) at $550^{\circ} \mathrm{C}$ for 24 hours. The ash samples were taken from the furnace and allowed to cool in desiccators provided with a desiccant agent. After cooling, the samples were weighed and replaced in the oven until the differences between two successive weightings were not exceeded $\pm 0.001 \mathrm{~g}$. Acid digestion method was used to determine elements constituents. Each sample $(2.0 \mathrm{~g})$ was digested with a mixture of $\mathrm{HNO}_{3}(0.1 \mathrm{M}, 20 \mathrm{~mL})$ and $\mathrm{HCl}(6 \mathrm{M}, 5 \mathrm{~mL})$ at the room temperature $\left(25^{\circ} \mathrm{C}\right)$ [29], [30]. The solution was subsequently transferred to a $50 \mathrm{~mL}$ volumetric flask and diluted with deionized water. The amounts of elements were analyzed using an ICP-OES. The analysis was performed in triplicate and the results were presented in mean value \pm standard deviation.

The concentration $(\mathrm{C})$ of the metal in the test sample was then calculated using the following formula:

$$
C=\frac{(a-b) x V}{m} \cdot d f
$$


where $C$ means concentration of elements in the test sample $(\mathrm{mg} / \mathrm{kg}), \quad a=$ concentration in the experiment solution $(\mathrm{mg} / \mathrm{mL}), b=$ mean concentration in the blank solutions $(\mathrm{mg} / \mathrm{mL}), V=$ volume of the experiment solution (mL), $m=$ weight of the test portion $(\mathrm{g})$ and $d f=$ dilution factor.

\section{Data Analysis}

Significance between elements was tested with one way ANOVA using Minitab statistical software (Version 16, Minitab Inc., USA). Differences among means were considered significant at $\mathrm{p}<0.05$. Multivariate data of major and minor elements in dates-flesh and dates-pits (triplicates samples) were mean-normalized using Microsoft Excel 2007. Exploratory analysis technique using PCA was performed on the normalized data matrix using Unscrambler 10.3 (CAMO software AS, Oslo, Norway) to identify the relationship between samples studied and its elements content in scores and loadings plots.

\section{RESULTS AND DISCUSSION}

\section{A. Analysis of Variance (ANOVA)}

ANOVA by one way technique on dataset of ICP-OES showed significant differences among samples in dates-flesh and dates-pits.

\section{B. Dates-Flesh}

The major and minor elements of ten varieties of dateflesh samples are presented in Table 1. The results showed significant $(p<0.05)$ differences among several of the 10 varieties.

\section{Major Elements}

The content of each sample in Table 1 showed that $\mathrm{K}, \mathrm{Ca}$, $\mathrm{Mg}$ and $\mathrm{Na}$ are higher $(95 \mathrm{mg} / \mathrm{kg}-5623 \mathrm{mg} / \mathrm{kg}$ ) than other components hence considered as the major elements while $\mathrm{Al}, \mathrm{Ba}, \mathrm{Cu}, \mathrm{Fe}, \mathrm{Mn}, \mathrm{Ni}, \mathrm{Pb}, \mathrm{Zn}$ and $\mathrm{Se}$ were grouped as minor elements (less than $52 \mathrm{mg} / \mathrm{kg}$ ), except for Al where the maximum value was $105.8 \mathrm{mg} / \mathrm{kg}$ in Ajwa date. $\mathrm{Pb}$ and $\mathrm{Cd}$ were the main heavy metals in dates due to rapidly increasing population in urban areas led to anthropogenic activities and fossil fuel combustion. Emissions from road traffic that uses fossil fuel, industry, agriculture, sewage sludge, and waste incineration are the chief sources of air pollution [31], [32]. The maximum permitted metals contaminant in fruit products in Malaysian Food Act 1983 are $\mathrm{Pb}(2 \mathrm{mg} / \mathrm{kg}), \mathrm{Cd}(1 \mathrm{mg} / \mathrm{kg}), \mathrm{As}(1 \mathrm{mg} / \mathrm{kg}), \mathrm{Hg}(0.05$ $\mathrm{mg} / \mathrm{kg})$ and $\mathrm{Sb}(1 \mathrm{mg} / \mathrm{kg})$ and therefore the levels of $\mathrm{Pb}$ in date-flesh $(0.090 \mathrm{mg} / \mathrm{kg}-0.534 \mathrm{mg} / \mathrm{kg})$ is within the permissible limit. $\mathrm{K}$ content of the samples was between $2823 \mathrm{mg} / \mathrm{kg}$ to $5623 \mathrm{mg} / \mathrm{kg}$, with the highest value recorded for Mabroom, and the lowest was observed in Medjool. The wide range of $\mathrm{K}$ was similar to those reported by [33] in the range of $3500 \mathrm{mg} / \mathrm{kg}$ to $7500 \mathrm{mg} / \mathrm{kg}$ in dates from different varieties. $\mathrm{K}$ is known as an essential nutrient in maintaining the nervous system [29]. Lack of $\mathrm{K}$ in body may lead to hypokalemia and causes elevation in blood pressure [34]. $\mathrm{Mg}$ concentration of Nourina, Mesir, Mariami and Medjool was in the range of $507 \mathrm{mg} / \mathrm{kg}$ to $530 \mathrm{mg} / \mathrm{kg}$ higher than those recorded for other samples that ranged from 336 $\mathrm{mg} / \mathrm{kg}$ to $493 \mathrm{mg} / \mathrm{kg}$. Sunsweet dates registered the lowest

content of $\mathrm{Mg}(336 \mathrm{mg} / \mathrm{kg})$. The evaluated $\mathrm{Mg}$ concentration of the date samples was within the range and comparable to the $\mathrm{Mg}$ content in date fruits from different origins as reported by others. In [29] reported the concentration of $\mathrm{Mg}$ in dates was $480 \mathrm{mg} / \mathrm{kg}$ to $530 \mathrm{mg} / \mathrm{kg}$; in [33] mentioned that the $\mathrm{Mg}$ concentration of their date samples was 262 $\mathrm{mg} / \mathrm{kg}$ to $2250 \mathrm{mg} / \mathrm{kg}$, while in [35] reported $\mathrm{Mg}$ concentration of $560 \mathrm{mg} / \mathrm{kg}$ to $600 \mathrm{mg} / \mathrm{kg}$. Variability in Ca concentration among the date samples was recorded with values ranging from $343 \mathrm{mg} / \mathrm{kg}$ to $870 \mathrm{mg} / \mathrm{kg}$. Ajwa dates recorded highest $\mathrm{Ca}$ concentration $(870 \mathrm{mg} / \mathrm{kg})$ followed by Mariami dates $(707 \mathrm{mg} / \mathrm{kg})$. The lowest $\mathrm{Ca}$ content was recorded for Mesir date $(343 \mathrm{mg} / \mathrm{kg})$. The Ca concentration of the studied dates was comparable to the dates from previous studies. In [33] reported Ca concentration of 249 $\mathrm{mg} / \mathrm{kg}$ to $1100 \mathrm{mg} / \mathrm{kg}$. In 29] reported Ca concentration of $480 \mathrm{mg} / \mathrm{kg}$ to $530 \mathrm{mg} / \mathrm{kg}$, while in [36] reported Ca concentration of $12 \mathrm{mg} / \mathrm{kg}$ to $800 \mathrm{mg} / \mathrm{kg}$. In contrast, higher concentration of $\mathrm{Ca}$ in dates was recorded by [19] with values $1230 \mathrm{mg} / \mathrm{kg}$ to $1870 \mathrm{mg} / \mathrm{kg}$ and in [35] reported Ca concentration of $1330 \mathrm{mg} / \mathrm{kg}$ to $2070 \mathrm{mg} / \mathrm{kg}$ for dates from different countries. Ca works together with $\mathrm{Mg}$ and help in bone development and energy metabolism [19]. $\mathrm{Na}$ concentration of 10 samples was in the range between 95 $\mathrm{mg} / \mathrm{kg}$ for Khudry to $473 \mathrm{mg} / \mathrm{kg}$ for Ajwa dates. People who are suffering from hypertension are advisable to consume dates due to its high $\mathrm{K}$ and low $\mathrm{Na}$ content.

\section{Minor Elements}

Limited information reported on the $\mathrm{Al}$ content in date fruit, which it can be described as a heavy metal. Trace elements and heavy metals have certain health benefits at lower concentrations, but at higher levels, they can be toxic and pose health risks [14]. The European Food Safety Authority (EFSA) has established for the lifelong intake of $\mathrm{Al}$ at a tolerable weekly intake (TWI) of $1 \mathrm{mg} / \mathrm{kg}$ body weight [36]. Al was detected present in the samples in the range of $16.5 \mathrm{mg} / \mathrm{kg}$ to $106 \mathrm{mg} / \mathrm{kg}$. Ajwa dates were recorded to contain $106 \mathrm{mg} / \mathrm{kg}$ and significantly $(\mathrm{p}<0.05)$ higher than other dates samples. Medjool dates contain 51 $\mathrm{mg} / \mathrm{kg}$ and Mariami contain $48 \mathrm{mg} / \mathrm{kg}$ of $\mathrm{Al}$. Low $\mathrm{Al}$ concentration was recorded for Khudry $(16.5 \mathrm{mg} / \mathrm{kg})$ similar to that reported by [37] which was in the range of 15.2 $\mathrm{mg} / \mathrm{kg}$ to $16.7 \mathrm{mg} / \mathrm{kg}$. The Ba concentration of date samples ranged between $0.115 \mathrm{mg} / \mathrm{kg}$ to $0.86 \mathrm{mg} / \mathrm{kg}$ and Ajwa dates recorded significantly $(\mathrm{p}<0.05)$ high $\mathrm{Ba}$ content $(0.86$ $\mathrm{mg} / \mathrm{kg}$ ), while other samples were in the range between $0.115 \mathrm{mg} / \mathrm{kg}$ to $0.45 \mathrm{mg} / \mathrm{kg}$. Ba compound is widely used in industrial application such as in the production of plastics, textile and rubber [38]. Ba can be found naturally in drinking water and food, usually at low levels but it is not considered to be an essential element for human health. In [39] reported maximum daily dietary intake of barium for adults as $0.72 \mathrm{mg} /$ person. The concentration of $\mathrm{Ba}$ in date samples is within the permitted range as humans consume small amount of dates per day, example Ba content in $200 \mathrm{~g}$ of Ajwa dates is only $0.172 \mathrm{mg}$. $\mathrm{Cu}$ is vital for human body, 


\section{ASSESSMENT OF MINERALS IN PHOENIX DACTYLIFERA L. AS DETERMINED BY INDUCTIVELY COUPLED PLASMA OPTICAL EMISSION SPECTROMETRY USING ANOVA AND PCA}

especially for the nervous and cardiovascular systems. The obtained results was in the range value of $0.7 \mathrm{mg} / \mathrm{kg}-7.7$ $\mathrm{mg} / \mathrm{kg}$ and $2.7 \mathrm{mg} / \mathrm{kg}-7.9 \mathrm{mg} / \mathrm{kg}$ as reported by [33], [40] respectively, but higher levels was reported by [41] (12 $\mathrm{mg} / \mathrm{kg}-727 \mathrm{mg} / \mathrm{kg})$ and in [42] $(8.9 \mathrm{mg} / \mathrm{kg}-18.6 \mathrm{mg} / \mathrm{kg})$. Maximum concentration values in $\mathrm{Cu}$ were obtained in Medjool $(6.23 \mathrm{mg} / \mathrm{kg}$ ) and Khudry $(5.88 \mathrm{mg} / \mathrm{kg})$ samples, while minimum concentrations of $\mathrm{Cu}$ were noted in DegletNour (1.86 mg/kg) and Mabroom $(2.98 \mathrm{mg} / \mathrm{kg})$ samples. The tolerable upper intake level for adults is 10 $\mathrm{mg} /$ day [43]. The concentration of $\mathrm{Cu}$ in date samples is within the permissible range. Iron deficiency is considered as the number one nutritional disorder in the world by the World Health Organization (WHO).

Table 1: The elements concentration of dates-flesh and pits

\begin{tabular}{|c|c|c|c|c|c|c|c|c|c|c|c|c|c|c|c|c|c|c|c|c|c|c|c|c|c|c|}
\hline \multirow[t]{3}{*}{ Sample } & \multicolumn{26}{|c|}{ Concentration of Dates $(\mathrm{mg} / \mathrm{kg})$} \\
\hline & \multicolumn{2}{|c|}{$\mathrm{Al}$} & \multicolumn{2}{|c|}{$\mathrm{Ba}$} & \multicolumn{2}{|c|}{$\mathrm{Ca}$} & \multicolumn{2}{|c|}{$\mathrm{Cu}$} & \multicolumn{2}{|c|}{$\mathrm{Fe}$} & \multicolumn{2}{|c|}{$\mathrm{Mg}$} & \multicolumn{2}{|c|}{$\mathrm{Mn}$} & \multicolumn{2}{|c|}{$\mathrm{Na}$} & \multicolumn{2}{|c|}{$\mathrm{Ni}$} & \multicolumn{2}{|c|}{$\mathrm{Pb}$} & \multicolumn{2}{|c|}{$\mathrm{Zn}$} & \multicolumn{2}{|c|}{$\mathrm{Se}$} & \multicolumn{2}{|c|}{ K } \\
\hline & Flesh & Pit & Flesh & Pit & Flesh & Pit & Flesh & Pit & Flesh & Pit & Flesh & Pit & Flesh & Pit & Flesh & Pit & Flesh & Pit & Flesh & Pit & Flesh & Pits & Flesh & Pit & Flesh & PPit \\
\hline Sunsweet & $\begin{array}{c}26.5^{\mathrm{d}} \\
\pm \\
1.27\end{array}$ & $\begin{array}{c}6.96^{\mathrm{b}} \\
\pm \\
0.80\end{array}$ & $\begin{array}{c}0.259^{\mathrm{c}} \\
\mathrm{d} \\
\pm \\
0.05\end{array}$ & $\begin{array}{l}0.139^{\mathrm{b}} \\
\pm 0.01\end{array}$ & \begin{tabular}{|c|}
$488^{\mathrm{de}}$ \\
\pm \\
\pm \\
23.6 \\
5 \\
\end{tabular} & $\begin{array}{c}186^{\mathrm{f}} \\
\pm \\
\pm \\
1.96\end{array}$ & $\begin{array}{c}4.47^{\mathrm{b}} \\
\pm \\
1.03\end{array}$ & $\mid \begin{array}{c}22.57^{\mathrm{c}} \\
\mathrm{d} \pm \\
\pm \\
1.15\end{array}$ & $\begin{array}{c}8.08^{\mathrm{e}} \pm \\
0.17\end{array}$ & $\begin{array}{c}13.18 \\
\mathrm{~b} \pm \\
0.53\end{array}$ & \begin{tabular}{|c|}
$336^{\mathrm{e}}$ \\
\pm \\
11.7 \\
3
\end{tabular} & \begin{tabular}{|c|}
$557^{\mathrm{de}}$ \\
\pm \\
16.1 \\
3 \\
\end{tabular} & $\begin{array}{c}3.36^{b} \\
\pm \\
0.87\end{array}$ & $\begin{array}{c}8.90^{\mathrm{b}} \\
\pm \\
0.13\end{array}$ & $\mid \begin{array}{c}123^{\mathrm{ef}} \\
\pm \\
0.60\end{array}$ & $\mid \begin{array}{c}137 \\
\mathrm{~d} \pm \\
\pm .52\end{array}$ & $\begin{array}{c}0.194^{\mathrm{fg}} \\
\pm 0.03\end{array}$ & $\begin{array}{c}3.10^{\mathrm{b}} \\
\pm 0.50\end{array}$ & $\begin{array}{c}0.484^{\mathrm{a}} \\
\mathrm{b} \pm \\
0.06\end{array}$ & $\left|\begin{array}{c}1.27^{b} \\
\pm \\
0.07\end{array}\right|$ & $\mid \begin{array}{c}3.28^{\mathrm{c}} \\
\pm \\
0.18\end{array}$ & $\begin{array}{c}22.30 \\
\mathrm{~d} \\
\mathbf{\pm} \\
0.74\end{array}$ & $\left|\begin{array}{c}0.138 \\
\mathrm{a} \pm \\
0.02\end{array}\right|$ & ND & $\begin{array}{c}3612^{\mathrm{d}} \\
\mathrm{e} \\
\pm \\
57.8\end{array}$ & $\begin{array}{l}1249^{\mathrm{de}} \\
\pm 74.5\end{array}$ \\
\hline Nourina & $\begin{array}{c}43.3^{\mathrm{bc}} \\
\pm \\
4.50 \\
\end{array}$ & $\begin{array}{c}3.05^{\mathrm{cd}} \\
\pm \\
0.46\end{array}$ & $\begin{array}{c}0.356^{b} \\
{ }^{c} \pm \\
0.04\end{array}$ & $\begin{array}{l}0.131^{\mathrm{b}} \\
\pm 0.03\end{array}$ & $\begin{array}{c}554^{\mathrm{cd}} \\
\pm \\
0.69\end{array}$ & $\left|\begin{array}{c}208^{d} \\
e^{ \pm} \\
5.75\end{array}\right|$ & $\begin{array}{c}4.31^{\mathrm{b}} \\
\pm \\
0.17\end{array}$ & $\begin{array}{l}12.39^{\mathrm{e}} \\
\pm 1.48\end{array}$ & $\begin{array}{l}10.56^{\mathrm{cd}} \\
\pm 1.25\end{array}$ & \begin{tabular}{c|}
12.32 \\
$\mathrm{~b} \pm$ \\
1.23
\end{tabular} & $\begin{array}{c}507^{\mathrm{ab}} \\
\pm \\
41.5 \\
0\end{array}$ & \begin{tabular}{|c|}
$629^{b}$ \\
\pm \\
35.0 \\
2 \\
\end{tabular} & $\begin{array}{c}3.32^{\mathrm{b}} \\
\pm \\
0.27\end{array}$ & $\begin{array}{c}9.66^{\mathrm{b}} \\
\pm \\
0.57\end{array}$ & $\begin{array}{c}247^{\mathrm{c}} \\
\pm \\
26.7 \\
7\end{array}$ & $\left|\begin{array}{c}113^{\mathrm{e}} \\
\pm \\
4.76\end{array}\right|$ & $\begin{array}{c}0.273^{\mathrm{de}} \\
\pm 0.02\end{array}$ & \begin{tabular}{|l|}
$1.83^{\mathrm{de}}$ \\
\pm 0.27
\end{tabular} & $\begin{array}{c}0.488^{\mathrm{a}} \\
\mathrm{b} \pm \\
0.04\end{array}$ & $\left|\begin{array}{c}0.67^{\mathrm{d}} \\
\pm \\
0.002\end{array}\right|$ & $\mid \begin{array}{c}3.28^{c} \\
\pm \\
0.17\end{array}$ & $\mid \begin{array}{c}13.41 \\
\mathrm{e} \pm \\
0.82\end{array}$ & ND & ND & $\begin{array}{c}5185^{\mathrm{a}} \\
\mathrm{b} \pm \\
29.6\end{array}$ & $\begin{array}{l}1149^{\mathrm{e}} \\
\pm 40.2\end{array}$ \\
\hline Mabroom & \begin{tabular}{|c|}
$24.1^{\mathrm{de}}$ \\
\pm \\
3.08 \\
\end{tabular} & $\begin{array}{c}4.19^{\mathrm{cd}} \\
\pm \\
0.09 \\
\end{array}$ & \begin{tabular}{|c}
$0.172^{\mathrm{d}}$ \\
$\mathrm{e} \pm$ \\
0.001
\end{tabular} & $\begin{array}{l}0.138^{b} \\
\pm 0.01\end{array}$ & $\begin{array}{c}483^{\mathrm{de}} \\
\pm \\
2.55 \\
\end{array}$ & $\begin{array}{c}260^{b} \\
\pm \\
2.17 \\
\end{array}$ & $\begin{array}{c}2.98^{c} \\
d \\
\pm \\
0.36 \\
\end{array}$ & $\begin{array}{l}20.89^{\mathrm{d}} \\
\pm 1.11 \\
\end{array}$ & $\begin{array}{l}9.76^{\mathrm{de}} \\
\pm 0.91\end{array}$ & \begin{tabular}{|c|}
11.17 \\
$\mathrm{~b} \pm$ \\
0.19 \\
\end{tabular} & $\begin{array}{c}423^{\mathrm{cd}} \\
\pm \\
1.60 \\
\end{array}$ & \begin{tabular}{|c|}
$586^{\mathrm{cd}}$ \\
\pm \\
7.01 \\
\end{tabular} & $\begin{array}{c}1.37^{\mathrm{d}} \\
\pm \\
0.02 \\
\end{array}$ & \begin{tabular}{|c|}
$6.87^{c}$ \\
\pm \\
0.14 \\
\end{tabular} & \begin{tabular}{|c|}
$139^{\mathrm{e}}$ \\
\pm \\
4.37 \\
\end{tabular} & $\begin{array}{c}133 \\
\mathrm{~d} \\
\pm \\
5.42\end{array}$ & $\begin{array}{l}0.500^{\mathrm{a}} \\
\pm 0.04\end{array}$ & \begin{tabular}{|c|}
$2.25^{\mathrm{bc}}$ \\
$\mathrm{d} \pm$ \\
0.18 \\
\end{tabular} & $\begin{array}{l}0.534^{\mathrm{a}} \\
\pm 0.12 \\
\end{array}$ & \begin{tabular}{|c|}
$1.05^{\mathrm{c}}$ \\
\pm \\
0.07 \\
\end{tabular} & \begin{tabular}{|c|}
$3.79^{b}$ \\
$c \pm$ \\
0.19 \\
\end{tabular} & \begin{tabular}{|c|}
$\begin{array}{c}21.09 \\
\mathrm{~d} \\
\pm \\
0.36\end{array}$ \\
\end{tabular} & $\begin{array}{c}0.065 \\
\mathrm{a} \pm \\
0.09 \\
\end{array}$ & ND & \begin{tabular}{|c|}
$5623^{\mathrm{a}}$ \\
\pm \\
342.2 \\
\end{tabular} & $\begin{array}{r}1235^{\mathrm{e}} \\
\pm 14.1 \\
\end{array}$ \\
\hline Khudry & \begin{tabular}{|c|}
$16.5^{\mathrm{e}}$ \\
\pm \\
0.90 \\
\end{tabular} & \begin{tabular}{|c}
$4.32^{\mathrm{c}}$ \\
\pm \\
0.847 \\
\end{tabular} & $\begin{array}{l}0.115^{\mathrm{e}} \\
\pm 0.01 \\
\end{array}$ & $\begin{array}{l}0.116^{b} \\
\pm 0.01\end{array}$ & $\begin{array}{c}474^{\mathrm{de}} \\
\pm \\
5.86 \\
\end{array}$ & $\begin{array}{c}216^{\mathrm{d}} \\
\mathrm{e} \pm \\
2.66 \\
\end{array}$ & \begin{tabular}{|c|}
$5.88^{\mathrm{a}}$ \\
\pm \\
0.32 \\
\end{tabular} & \begin{tabular}{|l|}
$33.82^{\mathrm{b}}$ \\
\pm 1.42 \\
\end{tabular} & $\begin{array}{l}7.68^{e} \pm \\
0.23\end{array}$ & \begin{tabular}{|c|}
12.05 \\
$\mathrm{~b} \pm$ \\
0.35 \\
\end{tabular} & $\begin{array}{c}383^{\mathrm{de}} \\
\pm \\
9.52 \\
\end{array}$ & \begin{tabular}{|c|}
$538^{\mathrm{e}}$ \\
\pm \\
2.68 \\
\end{tabular} & $\begin{array}{c}2.85^{b} \\
\mathrm{c} \\
\pm \\
0.16 \\
\end{array}$ & \begin{tabular}{|c|}
$9.04^{b}$ \\
\pm \\
0.10 \\
\end{tabular} & $\begin{array}{l}95^{\mathrm{f}} \pm \\
1.81 \\
\end{array}$ & $\begin{array}{c}130 \\
\mathrm{~d} \pm \\
\pm \\
1.87\end{array}$ & $\begin{array}{l}0.418^{b} \\
\pm 0.03\end{array}$ & \begin{tabular}{|c|}
$6.15^{\mathrm{a}}$ \\
\pm 0.31 \\
\end{tabular} & $\begin{array}{c}0.418^{\mathrm{a}} \\
\mathrm{b} \pm \\
0.06 \\
\end{array}$ & \begin{tabular}{|c|}
$1.55^{\mathrm{a}}$ \\
\pm \\
0.03 \\
\end{tabular} & \begin{tabular}{|c|}
$4.27^{b}$ \\
\pm \\
0.28 \\
\end{tabular} & \begin{tabular}{|c|}
31.13 \\
$\mathrm{~b} \pm$ \\
0.45 \\
\end{tabular} & \begin{tabular}{|c|}
0.107 \\
$\mathrm{a} \pm$ \\
0.14 \\
\end{tabular} & ND & $\begin{array}{c}4594^{b} \\
{ }^{\mathrm{c}} \pm \\
266.2\end{array}$ & $\begin{array}{r}1908^{\mathrm{a}} \\
\pm 44.5 \\
\end{array}$ \\
\hline Sunseed & \begin{tabular}{|c|}
$41.2^{\mathrm{c}}$ \\
\pm \\
4.85 \\
\end{tabular} & $\begin{array}{c}3.57^{\mathrm{cd}} \\
\pm \\
0.44\end{array}$ & $\begin{array}{l}0.403^{b} \\
\pm 0.07\end{array}$ & $\begin{array}{c}0.151^{b} \\
\pm 0.003\end{array}$ & \begin{tabular}{|c|}
$419^{\mathrm{ef}}$ \\
\pm \\
10.8 \\
8 \\
\end{tabular} & $\begin{array}{c}226^{\mathrm{c}} \\
\mathrm{d} \pm \\
\pm \\
1.41\end{array}$ & $\begin{array}{c}4.18^{b} \\
{ }^{\mathrm{b}} \pm \\
0.11\end{array}$ & $\begin{array}{l}25.82^{\mathrm{c}} \\
\pm 2.20\end{array}$ & $\begin{array}{c}8.12^{\mathrm{e}} \pm \\
0.84\end{array}$ & \begin{tabular}{|c|}
13.72 \\
${ }^{b} \pm$ \\
0.36
\end{tabular} & $\begin{array}{c}392^{\mathrm{de}} \\
\pm \\
15.6 \\
5\end{array}$ & $\begin{array}{c}565^{\mathrm{de}} \\
\pm \\
9.38\end{array}$ & $\begin{array}{c}2.69^{b} \\
c \pm \\
0.53\end{array}$ & $\begin{array}{c}7.12^{\mathrm{c}} \\
\pm \\
0.23\end{array}$ & $\mid \begin{array}{c}219^{\mathrm{cd}} \\
\pm \\
7.55\end{array}$ & $\left|\begin{array}{c}127 \\
\mathrm{~d} \pm \\
\pm \\
1.70\end{array}\right|$ & $\begin{array}{c}0.354^{\mathrm{bc}} \\
\pm 0.03\end{array}$ & $\begin{array}{l}3.22^{\mathrm{b}} \\
\pm 0.18\end{array}$ & $\begin{array}{c}0.370^{\mathrm{a}} \\
\mathrm{b} \pm \\
0.04\end{array}$ & $\left|\begin{array}{c}1.27^{\mathrm{b}} \\
\pm \\
0.002\end{array}\right|$ & $\left|\begin{array}{c}3.74^{b} \\
{ }^{\mathrm{c}} \pm \\
0.25\end{array}\right|$ & $\left|\begin{array}{c}25.02 \\
{ }^{c} \pm \\
0.598\end{array}\right|$ & $\left|\begin{array}{c}0.066 \\
\mathrm{a} \pm \\
0.17\end{array}\right|$ & ND & $\left|\begin{array}{c}4441^{b} \\
{ }^{\mathrm{c}} \pm \\
366.9\end{array}\right|$ & $\begin{array}{c}1392^{\mathrm{bc}} \\
\mathrm{d} \pm \\
67.1\end{array}$ \\
\hline$\underset{\mathrm{r}}{\text { Degletnou }}$ & $\begin{array}{c}17.8^{\mathrm{e}} \\
\pm \\
0.89\end{array}$ & $\begin{array}{c}4.10^{\mathrm{cd}} \\
\pm \\
0.33\end{array}$ & $\begin{array}{c}0.147^{\mathrm{d}} \\
\mathrm{e} \\
\pm \\
0.03\end{array}$ & $\begin{array}{c}0.125^{\mathrm{b}} \\
\pm \\
0.0003\end{array}$ & \begin{tabular}{|c}
$558^{\mathrm{cd}}$ \\
\pm \\
27.6 \\
3 \\
\end{tabular} & $\begin{array}{c}188^{\mathrm{f}} \\
\pm \\
2.47 \\
\end{array}$ & $\begin{array}{c}1.86^{\mathrm{d}} \\
\pm \\
0.32\end{array}$ & $\begin{array}{l}21.20^{\mathrm{d}} \\
\pm 0.70\end{array}$ & $\begin{array}{l}7.52^{\mathrm{e}} \pm \\
0.28\end{array}$ & $\begin{array}{c}13.93 \\
\mathrm{~b} \pm \\
0.43\end{array}$ & $\begin{array}{c}454^{\mathrm{bc}} \\
\pm \\
10.0 \\
0 \\
\end{array}$ & \begin{tabular}{|c|}
$647^{\mathrm{b}}$ \\
\pm \\
10.7 \\
4 \\
\end{tabular} & $\begin{array}{c}3.08^{\mathrm{b}} \\
\pm \\
0.18\end{array}$ & \begin{tabular}{|c|}
$7.71^{c}$ \\
\pm \\
0.13 \\
\end{tabular} & $\begin{array}{c}189^{\mathrm{d}} \\
\pm \\
23.3 \\
0 \\
\end{array}$ & $\begin{array}{c}130 \\
\mathrm{~d} \pm \\
\mathbf{3} .91\end{array}$ & $\begin{array}{c}0.206^{\mathrm{ef}} \\
\mathrm{g}_{ \pm} \\
0.01\end{array}$ & $\begin{array}{l}2.37^{\mathrm{bc}} \\
\pm 0.36\end{array}$ & $\begin{array}{l}0.354^{\mathrm{b}} \\
\pm 0.06\end{array}$ & $\left|\begin{array}{c}1.25^{b} \\
\mathrm{c} \pm \\
0.02\end{array}\right|$ & $\begin{array}{c}2.42^{\mathrm{d}} \\
\pm \\
0.14\end{array}$ & $\left|\begin{array}{c}20.39 \\
d \pm \\
1.06\end{array}\right|$ & $\left|\begin{array}{c}0.074 \\
\mathrm{a} \pm \\
0.04\end{array}\right|$ & ND & $\mid \begin{array}{c}4298^{\mathrm{c}} \\
\mathrm{d}_{ \pm}^{ \pm} \\
287.3\end{array}$ & $\begin{array}{c}1389^{\mathrm{bc}} \\
\mathrm{d} \pm \\
71.3\end{array}$ \\
\hline Mesir & \begin{tabular}{|c|}
$42.6^{\mathrm{bc}}$ \\
\pm \\
0.38
\end{tabular} & $\begin{array}{c}3.58^{\mathrm{cd}} \\
\pm \\
0.04\end{array}$ & $\begin{array}{c}0.344^{\mathrm{b}} \\
\mathrm{c} \pm \\
0.02\end{array}$ & $\begin{array}{l}0.108^{\mathrm{bc}} \\
\pm 0.03\end{array}$ & $\begin{array}{c}343^{\mathrm{f}} \\
\pm \\
3.91\end{array}$ & $\mid \begin{array}{c}236^{\mathrm{c}} \\
\pm \\
\pm .02\end{array}$ & $\begin{array}{c}5.16^{\mathrm{a}} \\
\mathrm{b} \pm \\
0.10\end{array}$ & $\begin{array}{c}6.67^{\mathrm{f}} \pm \\
0.28\end{array}$ & $=\begin{array}{l}17.61^{\mathrm{a}} \\
\pm 0.34\end{array}$ & $\begin{array}{c}17.22 \\
\mathrm{~b} \pm \\
0.29\end{array}$ & \begin{tabular}{|c}
$517^{\mathrm{a}}$ \\
\pm \\
25.9 \\
0
\end{tabular} & $\begin{array}{c}623^{b} \\
\pm \\
6.10\end{array}$ & $\begin{array}{c}5.02^{\mathrm{a}} \\
\pm \\
0.29\end{array}$ & $\mid \begin{array}{c}10.87 \\
\mathrm{a} \pm \\
0.45\end{array}$ & $\mid \begin{array}{c}390^{\mathrm{b}} \\
\pm \\
9.03\end{array}$ & $\left|\begin{array}{c}154^{c} \\
\pm \\
2.95\end{array}\right|$ & $\begin{array}{c}0.312^{\mathrm{cd}} \\
\pm 0.01\end{array}$ & $\begin{array}{c}1.50^{\mathrm{e}} \\
\pm 0.04\end{array}$ & $\begin{array}{l}0.328^{b} \\
\pm 0.02\end{array}$ & ND & $\begin{array}{c}6.70^{\mathrm{a}} \\
\pm \\
0.33\end{array}$ & $\mid \begin{array}{c}13.25 \\
\mathrm{e} \pm \\
0.38\end{array}$ & ND & $\left|\begin{array}{c}0.037 \\
\mathrm{a} \pm \\
0.02\end{array}\right|$ & $\mid \begin{array}{c}3060^{\mathrm{ef}} \\
\pm \\
359.2\end{array}$ & $\begin{array}{c}1284^{\mathrm{cd}} \\
{ }^{\mathrm{E}} \pm \\
32.9\end{array}$ \\
\hline Ajwa & $\begin{array}{c}105.8 \\
{ }^{a} \pm \\
1.10\end{array}$ & $\begin{array}{c}15.13 \\
\mathrm{a} \pm \\
0.49\end{array}$ & $\begin{array}{l}0.859^{\mathrm{a}} \\
\pm 0.09\end{array}$ & $\begin{array}{l}0.233^{\mathrm{a}} \\
\pm 0.03\end{array}$ & \begin{tabular}{|c|}
$870^{\mathrm{a}}$ \\
\pm \\
69.2 \\
7 \\
\end{tabular} & $\begin{array}{c}284^{\mathrm{a}} \\
\pm \\
4.83\end{array}$ & $\begin{array}{c}4.99^{\mathrm{a}} \\
\mathrm{b} \pm \\
0.39\end{array}$ & $\begin{array}{c}8.04^{\mathrm{f}} \pm \\
0.44\end{array}$ & $=\begin{array}{c}16.43^{\mathrm{ab}} \\
\pm 1.40\end{array}$ & \begin{tabular}{|c|}
79.96 \\
$\mathrm{a} \pm$ \\
1.18
\end{tabular} & $\begin{array}{c}493^{\mathrm{ab}} \\
\pm \\
16.1 \\
9 \\
\end{array}$ & $\mid \begin{array}{c}717^{\mathrm{a}} \\
\pm \\
5.70\end{array}$ & $\begin{array}{c}2.95^{\mathrm{b}} \\
\pm \\
0.11\end{array}$ & $\begin{array}{c}9.03^{\mathrm{b}} \\
\pm \\
0.27\end{array}$ & $\begin{array}{c}473^{\mathrm{a}} \\
\pm \\
6.37\end{array}$ & $\left|\begin{array}{c}332^{\mathrm{a}} \\
\pm \\
4.36\end{array}\right|$ & $\begin{array}{c}0.190^{\mathrm{fg}} \\
\pm 0.01\end{array}$ & $\begin{array}{c}0.34^{\mathrm{f}} \\
\pm 0.05\end{array}$ & $\begin{array}{l}0.140^{c} \\
\pm 0.05\end{array}$ & $\left|\begin{array}{c}0.10^{\mathrm{e}} \\
\pm \\
0.00\end{array}\right|$ & $\left|\begin{array}{c}3.94^{b} \\
{ }^{\mathrm{c}} \pm \\
0.50\end{array}\right|$ & $\mid \begin{array}{c}11.84 \\
\mathrm{e} \pm \\
0.12\end{array}$ & $\mid \begin{array}{c}0.223 \\
\mathrm{a} \pm \\
0.24\end{array}$ & ND & $\left|\begin{array}{c}3873^{c} \\
\mathrm{~d}^{ \pm} \\
46.3\end{array}\right|$ & $\begin{array}{l}1432^{\mathrm{bc}} \\
\pm 42.7\end{array}$ \\
\hline Medjool & $\begin{array}{c}51.2^{\mathrm{b}} \\
\pm \\
3.26\end{array}$ & $\begin{array}{c}2.62^{\mathrm{d}} \\
\pm \\
0.49\end{array}$ & $\begin{array}{l}0.406^{b} \\
\pm 0.05\end{array}$ & \begin{tabular}{|c}
$0.050^{\mathrm{c}}$ \\
\pm \\
0.0000 \\
7
\end{tabular} & \begin{tabular}{|c|}
$582^{\mathrm{c}}$ \\
\pm \\
38.6 \\
6 \\
\end{tabular} & $\begin{array}{c}273^{\mathrm{a}} \\
\mathrm{b} \pm \\
14.4\end{array}$ & $\begin{array}{c}6.23^{\mathrm{a}} \\
\pm \\
0.18\end{array}$ & $\begin{array}{l}39.91^{\mathrm{a}} \\
\pm 1.21\end{array}$ & $\begin{array}{c}11.89^{\mathrm{cd}} \\
\pm 0.45\end{array}$ & $\begin{array}{c}11.41 \\
\mathrm{~b} \pm \\
0.29\end{array}$ & $\begin{array}{c}530^{\mathrm{a}} \\
\pm \\
18.3 \\
9\end{array}$ & $\begin{array}{c}614^{\mathrm{bc}} \\
\pm \\
3.04\end{array}$ & $\begin{array}{c}2.97^{b} \\
\pm \\
0.37\end{array}$ & $\begin{array}{c}5.88^{\mathrm{d}} \\
\pm \\
0.15\end{array}$ & $\begin{array}{c}240^{\mathrm{c}} \\
\pm \\
2.79\end{array}$ & $\mid$\begin{tabular}{c|c}
203 & \\
$\mathrm{~b} \pm$ & 0 \\
4.97 & $\mathrm{f}$
\end{tabular} & $\begin{array}{l}0.236^{\mathrm{de}} \\
\pm 0.02\end{array}$ & $\begin{array}{c}6.62^{\mathrm{a}} \\
\pm 0.32\end{array}$ & $\begin{array}{l}0.090^{c} \\
\pm 0.03\end{array}$ & $\left|\begin{array}{c}1.59^{\mathrm{a}} \\
\pm \\
0.21\end{array}\right|$ & $\left|\begin{array}{c}3.84^{\mathrm{b}} \\
{ }^{\mathrm{c}} \pm \\
0.20\end{array}\right|$ & $\left|\begin{array}{c}36.33 \\
\mathrm{a} \pm \\
1.35\end{array}\right|$ & $\left|\begin{array}{c}0.215 \\
\mathrm{a} \pm \\
0.03\end{array}\right|$ & $\left|\begin{array}{c}0.100 \\
\mathrm{a} \pm \\
0.07\end{array}\right|$ & $\left|\begin{array}{c}2823^{f} \\
\pm \\
150.6\end{array}\right|$ & $\begin{array}{l}1492^{\mathrm{b}} \\
\pm 53.2\end{array}$ \\
\hline Mariami & $\begin{array}{c}48.3^{\mathrm{bc}} \\
\pm \\
2.84\end{array}$ & $\begin{array}{c}6.61^{\mathrm{b}} \\
\pm \\
0.21\end{array}$ & $\begin{array}{l}0.455^{\mathrm{b}} \\
\pm 0.03\end{array}$ & $\begin{array}{l}0.077^{\mathrm{bc}} \\
\pm 0.000\end{array}$ & \begin{tabular}{|c|}
$707^{\mathrm{b}}$ \\
\pm \\
55.8 \\
6 \\
\end{tabular} & $\begin{array}{c}204^{\mathrm{ef}} \\
\pm \\
9.13 \\
\end{array}$ & $\begin{array}{c}5.04^{\mathrm{a}} \\
\mathrm{b} \pm \\
0.38\end{array}$ & $\begin{array}{c}7.33^{\mathrm{f}} \pm \\
0.52\end{array}$ & $=\begin{array}{c}13.48^{\mathrm{bc}} \\
\pm 2.49\end{array}$ & $\begin{array}{c}11.97 \\
\mathrm{~b} \\
\pm 0.61 \\
\end{array}$ & \begin{tabular}{|c|}
$522^{\mathrm{a}}$ \\
\pm \\
24.0 \\
1 \\
\end{tabular} & \begin{tabular}{|c|}
$543^{\mathrm{e}}$ \\
\pm \\
11.6 \\
8 \\
\end{tabular} & $\begin{array}{c}1.62^{c} \\
d \pm \\
0.005 \\
\end{array}$ & $\begin{array}{c}9.57^{\mathrm{b}} \\
\pm \\
0.51\end{array}$ & $\begin{array}{c}248^{\mathrm{c}} \\
\pm \\
25.0 \\
7 \\
\end{array}$ & $\begin{array}{c}125 \\
\mathrm{~d} \pm \\
4.25\end{array} \mid$ & $\begin{array}{l}0.149^{\mathrm{g}} \\
\pm 0.03\end{array}$ & $\mid \begin{array}{c}2.00^{\text {cd }} \\
\mathrm{e}_{ \pm} \\
0.22\end{array}$ & $\begin{array}{l}0.099^{c} \\
\pm 0.04\end{array}$ & ND & $\left|\begin{array}{c}3.41^{b} \\
{ }^{b} \pm \\
0.17\end{array}\right|$ & $\begin{array}{c}11.58 \\
\mathrm{e} \pm \\
0.23\end{array}$ & $\mid \begin{array}{c}0.333 \\
\mathrm{a} \pm \\
0.05\end{array}$ & $\left|\begin{array}{c}0.125 \\
\mathrm{a} \pm \\
0.04\end{array}\right|$ & $\left|\begin{array}{c}5457^{\mathrm{a}} \\
\pm \\
348.5\end{array}\right|$ & $\begin{array}{l}1440^{\mathrm{b}} \\
\pm 57.3\end{array}$ \\
\hline
\end{tabular}

The Fe concentration of Mesir and Ajwa were 17.61 $\mathrm{mg} / \mathrm{kg}$ and $16.42 \mathrm{mg} / \mathrm{kg}$, respectively and higher than the $\mathrm{Fe}$ content of Sunseed $(8.12 \mathrm{mg} / \mathrm{kg})$, Sunsweet $(8.08 \mathrm{mg} / \mathrm{kg})$, Khudry $(7.68 \mathrm{mg} / \mathrm{kg}$ ) and DegletNour $(7.52 \mathrm{mg} / \mathrm{kg})$. The results of $\mathrm{Fe}$ concentration were within the range reported by others. In [33] reported the Fe content of $2.1 \mathrm{mg} / \mathrm{kg}-16.2$ $\mathrm{mg} / \mathrm{kg}$, while in [34] reported the Fe content of $8.5 \mathrm{mg} / \mathrm{kg}$ $72 \mathrm{mg} / \mathrm{kg}$. In contrast, higher Fe content of $40.6 \mathrm{mg} / \mathrm{kg}-69.1$ $\mathrm{mg} / \mathrm{kg}$ [41] and $32.1 \mathrm{mg} / \mathrm{kg}-52.6 \mathrm{mg} / \mathrm{kg}$ [35] were observed in dates from different countries. Mn concentrations of date samples ranged between $1.37 \mathrm{mg} / \mathrm{kg}$ and $5.02 \mathrm{mg} / \mathrm{kg}$. High Mn content was recorded for Egypt dates, and the lowest Mn concentration was observed in Mabroom dates. The Mn concentration in the studied samples was comparable with the previous reports. Mn concentration in ranges of 1.0 $\mathrm{mg} / \mathrm{kg}-7.0 \mathrm{mg} / \mathrm{kg}$ and $3 \mathrm{mg} / \mathrm{kg}-59 \mathrm{mg} / \mathrm{kg}$ were reported by [33], [43] respectively. In contrast, other studies reported higher concentrations of $\mathrm{Mn}$ in dates from various countries [10], [35], [40]. Mn is a crucial trace element for brain function and metabolism of protein and carbohydrates [10], [30]. High value of $\mathrm{Ni}$ in dates fruit was reported by [11] with the values in the range between $16.27 \mathrm{mg} / \mathrm{kg}$ and 17.85 $\mathrm{mg} / \mathrm{kg}$. In contrast, the result obtained in this study showed that $\mathrm{Ni}$ was present in very low concentrations in all the date varieties with values in range from $0.149 \mathrm{mg} / \mathrm{kg}$ (Mariami) to $0.499 \mathrm{mg} / \mathrm{kg}$ (Mabroom). The results are within the range of $0.071 \mathrm{mg} / \mathrm{kg}$ to $0.7 \mathrm{mg} / \mathrm{kg}$ as reported by [33]. The highest value of $\mathrm{Zn}$ was determined in Mesir dates with the concentration of $6.70 \mathrm{mg} / \mathrm{kg}$ and insignificant different noted between other samples in the range between 3.28 $\mathrm{mg} / \mathrm{kg}$ and $4.27 \mathrm{mg} / \mathrm{kg}$. Values of $\mathrm{Zn}$ obtained in this study were in agreement with the previous studies [33], [40], [42]. $\mathrm{Zn}$ is classified as an essential component which plays a vital role in sustaining proper immune function. Level of Se in all date samples were insignificantly different between ranges $0.065 \mathrm{mg} / \mathrm{kg}$ to $0.333 \mathrm{mg} / \mathrm{kg}$ and no Se was detected for Mesir and Nourina dates. According to [44] in their finding, Se is one of the elements found in dates and has a potential as anti-cancer and important in immune function.

Published By: 


\section{E. Dates-Pit}

\section{Major Elements}

The result from ICP-OES has revealed the concentration of minerals in dates-pit, as shown in Table 1. The major elements in dates-pit are same as flesh, but the values are less; K (1149 mg/kg-1908 mg/kg), Ca (186 mg/kg-284 $\mathrm{mg} / \mathrm{kg}), \mathrm{Mg}(538 \mathrm{mg} / \mathrm{kg}-717 \mathrm{mg} / \mathrm{kg})$ and $\mathrm{Na}(113 \mathrm{mg} / \mathrm{kg}-$ $203 \mathrm{mg} / \mathrm{kg}$ ). $\mathrm{Mg}$ was found to be higher than the dates-flesh. Khudry dates contained $1908 \mathrm{mg} / \mathrm{kg}$ of $\mathrm{K}$ and significantly $(\mathrm{p}<0.05)$ higher than other dates samples. Both Medjool and Mariami contain $1492 \mathrm{mg} / \mathrm{kg}$ and $1440 \mathrm{mg} / \mathrm{kg}$ of $\mathrm{K}$, respectively and insignificantly different. The $\mathrm{K}$ was noted lower in both Mabroom (1235 mg/kg) and Nourina (1149 $\mathrm{mg} / \mathrm{kg}$ ) samples. Variability in $\mathrm{Mg}$ concentration among dates samples were recorded with values ranging from 538 $\mathrm{mg} / \mathrm{kg}$ to $717 \mathrm{mg} / \mathrm{kg}$. Ajwa dates recorded highest $\mathrm{Mg}$ concentration $(717 \mathrm{mg} / \mathrm{kg})$ followed by DegletNour (647 $\mathrm{mg} / \mathrm{kg}$ ), Nourina $(629 \mathrm{mg} / \mathrm{kg}$ ) and Mesir samples (623 $\mathrm{mg} / \mathrm{kg}$ ). Meanwhile, the lowest $\mathrm{Mg}$ content was recorded for Khudry date $(538 \mathrm{mg} / \mathrm{kg})$. Na in dates-pit is usually slightly higher than $\mathrm{Ca}$, Ajwa has proven to have the maximum concentrations in both $\mathrm{Na}(332 \mathrm{mg} / \mathrm{kg})$ and $\mathrm{Ca}(284 \mathrm{mg} / \mathrm{kg})$. Nourina sample showed the lowest level of $\mathrm{Na}(113 \mathrm{mg} / \mathrm{kg})$, while Sunsweet and Deglet Nour were proven to have lower concentration $\mathrm{Ca}(186 \mathrm{mg} / \mathrm{kg})$. There is lack of information on the elemental compositions in other literature for datespit. In the United States, date pits have been a problem to the date industry as a waste stream. Pulverized ground date pits are being used on a small scale, on dirt roads as a type of road base gravel. However, finding a way to make a profit on the pits would benefit date farmers substantially [45].

\section{Minor Elements}

The minor elements such as $\mathrm{Fe}, \mathrm{Cu}, \mathrm{Zn}, \mathrm{Mn}, \mathrm{Pb}, \mathrm{Ni}$ and Se of dates pit for the 10 varieties are presented in Table 1. Fe value was the highest, followed by $\mathrm{Cu}, \mathrm{Zn}, \mathrm{Mn}, \mathrm{Pb}$ and $\mathrm{Ni}$. Fe concentration ranges between $11.17 \mathrm{mg} / \mathrm{kg}$ and 79.96 $\mathrm{mg} / \mathrm{kg}$ with the highest value observed in sample Ajwa, while no significant difference was observed in the others sample. $\mathrm{Cu}$ ranged between $6.67 \mathrm{mg} / \mathrm{kg}$ and $39.93 \mathrm{mg} / \mathrm{kg}$; Mesir had the lowest level of $\mathrm{Cu}$ while Medjool showed the highest level of $\mathrm{Cu}$. There is no significant difference in $\mathrm{Cu}$ content between Sunseed, Sunsweet, DegletNour and Mabroom. Ajwa, Mariami and Mesir are among the samples with the lower $\mathrm{Cu}$ content. The analysis showed highest level of $\mathrm{Zn}(36.3 \mathrm{mg} / \mathrm{kg})$ was present in sample Medjool and lowest level $(11.58 \mathrm{mg} / \mathrm{kg})$ was found in sample Mariami. ANOVA revealed insignificant difference in $\mathrm{Zn}$ content between samples Nourina, Mesir, Ajwa and Mariami which is in the range between $11.58 \mathrm{mg} / \mathrm{kg}$ and $13.41 \mathrm{mg} / \mathrm{kg}$. Ajwa showed the highest level of $\mathrm{Mn}(10.87 \mathrm{mg} / \mathrm{kg})$ and Medjool had the lowest content $(5.89 \mathrm{mg} / \mathrm{kg})$ while other samples were proven to have insignificant difference in the range of $6.87 \mathrm{mg} / \mathrm{kg}-7.71 \mathrm{mg} / \mathrm{kg}$ (Mabroom, Sunseed and DegletNour) and $8.9 \mathrm{mg} / \mathrm{kg}-9.66 \mathrm{mg} / \mathrm{kg}$ (Sunsweet, Ajwa, Khudry, Mariami and Nourina). Se was detected to be insignificantly different between concentration $0.037 \mathrm{mg} / \mathrm{kg}$ to $0.125 \mathrm{mg} / \mathrm{kg}$ for samples Mesir, Medjool and Marimi while the presence of Se was not detected in other samples.
Other minor elements that were observed higher in decreasing order are $\mathrm{Al}, \mathrm{Pb}, \mathrm{Ni}$ and $\mathrm{Ba}$. $\mathrm{Al}$ ranged between $2.62 \mathrm{mg} / \mathrm{kg}$ and $15.13 \mathrm{mg} / \mathrm{kg}$; Ajwa sample showed the highest level of $\mathrm{Al}(15.13 \mathrm{mg} / \mathrm{kg})$ and significantly different compared to other samples while the lowest value of $\mathrm{Al}$ was observed in Medjool sample (2.62 mg/kg). Ba levels ranged between $0.05 \mathrm{mg} / \mathrm{kg}$ and $0.23 \mathrm{mg} / \mathrm{kg}$ with Ajwa contains the highest level and other samples were proven to be insignificantly different with range $0.05 \mathrm{mg} / \mathrm{kg}$ to 0.15 $\mathrm{mg} / \mathrm{kg}$. The content of $\mathrm{Pb}$ in dates-pit was in the range of $0.10 \mathrm{mg} / \mathrm{kg}$ to $1.58 \mathrm{mg} / \mathrm{kg}$, which is within the permissible level. The highest level of $\mathrm{Pb}$ was found in Medjool while Ajwa shown the lowest value. $\mathrm{Pb}$ was not detected in Mariami and Mesir samples. Ni level was found to be highest in Medjool $(6.62 \mathrm{mg} / \mathrm{kg})$ and lowest in Ajwa $(0.34$ $\mathrm{mg} / \mathrm{kg})$.

\section{Principal Components Analysis (PCA)}

The similarities of dates-flesh and dates-pit for major and minor elements in Table 1 were well observed using Principal Components Analysis (PCA).

\section{F. Dates-Flesh}

\section{Major Elements}

The first principal component (PC1) and second principal component (PC2) accounted $90 \%$ and $7 \%$ of variance, respectively, was obtained after PCA was performed on the data matrix for major elements in dates-flesh samples (Table 1). The scores and loadings matrices obtained from PCA were used to create scores and loading plots of PC1 versus PC2 of the major elements in dates-flesh as shown in Fig. 1. Scores plot explains the distribution among the samples whereas the loadings plot explains the effect of elements on the distribution of samples. Samples that are closed together in scores plot have the similarities. The scores plot shows that Ajwa, Medjool and Mesir are located between 0.6-1.6 on the right side of $\mathrm{PC} 1$ while in loadings plot, the element of $\mathrm{Ca}, \mathrm{Mg}$ and $\mathrm{Na}$ are located around 0.25 on the right side of PC1. Thus, this indicates that Ajwa, Medjool and Mesir are significantly different from other samples which contained high level of $\mathrm{Ca}, \mathrm{Mg}$ and $\mathrm{Na}$. The confirmation was made by referring the value $(\mathrm{mg} / \mathrm{kg}$ ) of each element in Table 1 where Ajwa (870, 493, 473), Medjool (583, 530, 240) and Mesir (343, 517,390) for $\mathrm{Ca}, \mathrm{Mg}$ and $\mathrm{Na}$, respectively. The same way can be used to determine the composition of other samples for K. It can be seen that other samples are located on the left side of PC1 in the scores plot especially Mabroom (-1.2) where it can be correlated with the high level of $\mathrm{K}$ in the loadings plot. Mabroom and Sunsweet contained $5623 \mathrm{mg} / \mathrm{kg}$ and $3612 \mathrm{mg} / \mathrm{kg}$ of $\mathrm{K}$, respectively. Composition of $\mathrm{K}$ in Medjool and Mesir were found to be lower than Sunsweet but not for Ajwa where it recorded $3873 \mathrm{mg} / \mathrm{kg}$ which is slightly higher than Sunsweet. Mariami has high values of $\mathrm{Ca}(707 \mathrm{mg} / \mathrm{kg})$ and $\mathrm{Mg}(522 \mathrm{mg} / \mathrm{kg})$, however it is classified in the group which contain highest amount of K $(5457 \mathrm{mg} / \mathrm{kg})$. 


\section{ASSESSMENT OF MINERALS IN PHOENIX DACTYLIFERA L. AS DETERMINED BY INDUCTIVELY COUPLED PLASMA OPTICAL EMISSION SPECTROMETRY USING ANOVA AND PCA}

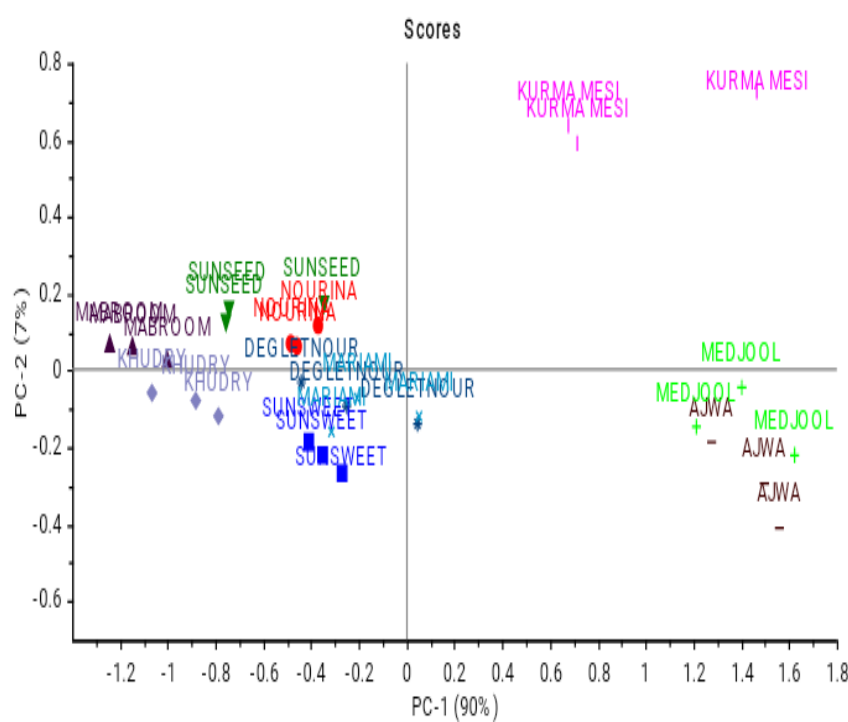

(a)

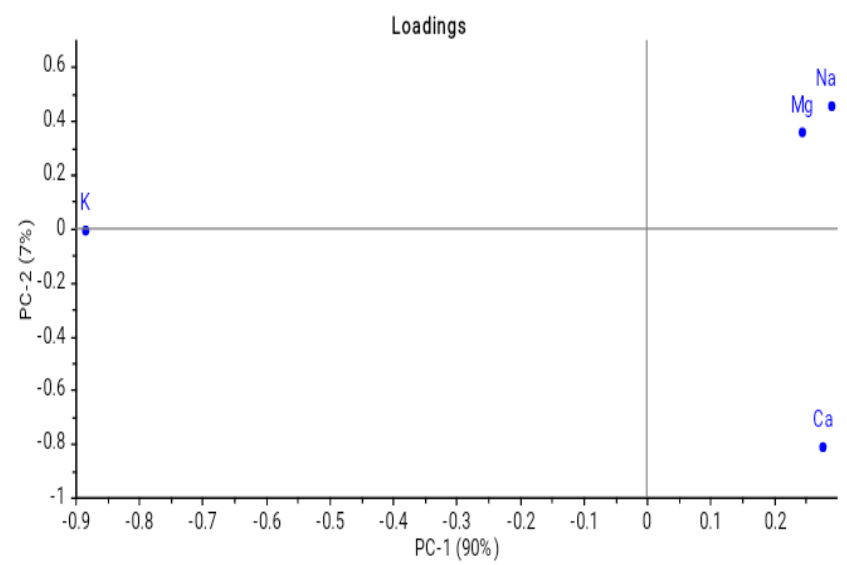

(b)

Fig. 1: Major elements plot of the first principal components (PC1) versus the second principal component (PC2) for the triplicate of 10 dates -flesh samples: (a) scores plot and (b) loadings plot

\section{Minor Elements}

Similarly, the scores and loadings matrices obtained from PCA were used to create scores and loading plots of PC1 (97\% variance) versus PC2 (2\% variance) of the minor elements in dates-flesh as shown in Fig. 2. Ajwa, Medjool, Mesir and Sunseed are distributed on the positive values of $\mathrm{PC} 1$ in the scores plot. Loadings plot shows that $\mathrm{Al}$ and $\mathrm{Fe}$ located at approximately 1 and 0.15 , respectively on PC1. Thus, it can be concluded that the samples contained high values $(\mathrm{mg} / \mathrm{kg})$ of $\mathrm{Al}$ and Fe; Ajwa (105.8, 16.43), Medjool (51.2, 11.89), Mesir $(42.6,17.61)$ and Sunseed $(41.2,8.12)$ respectively. However, it was observed that Sunseed is closely located with other samples due to its lowest value of Fe compared with Ajwa, Medjool and Mesir. Sunseed and other samples located at the center in the scores plot indicate that the contents of $\mathrm{Cu}, \mathrm{Zn}$ and $\mathrm{Mn}$; and followed by $\mathrm{Ba}, \mathrm{Ni}$, $\mathrm{Pb}$ and $\mathrm{Se}$ are closely similar. These were confirmed by referring to their values in Table 1 .

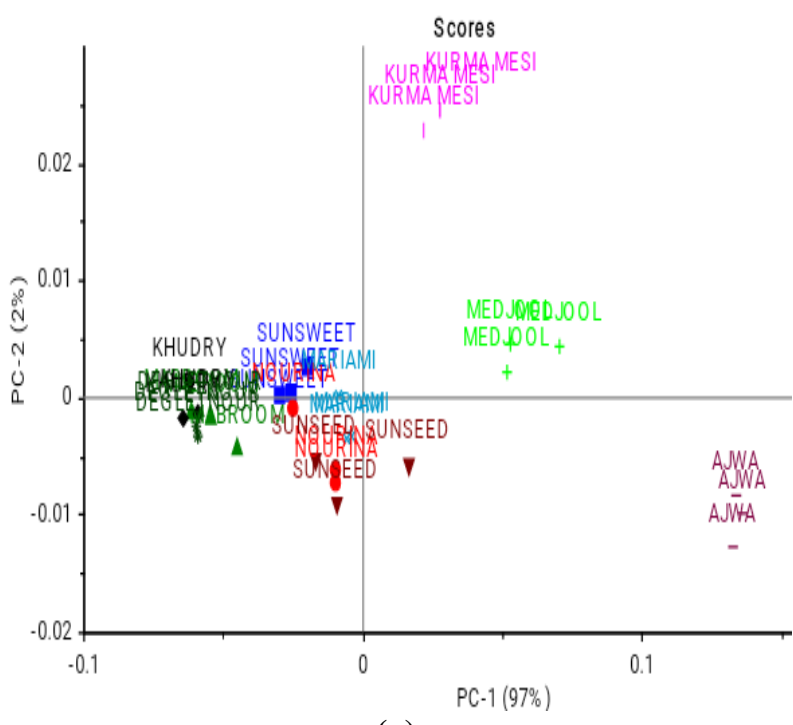

(a)

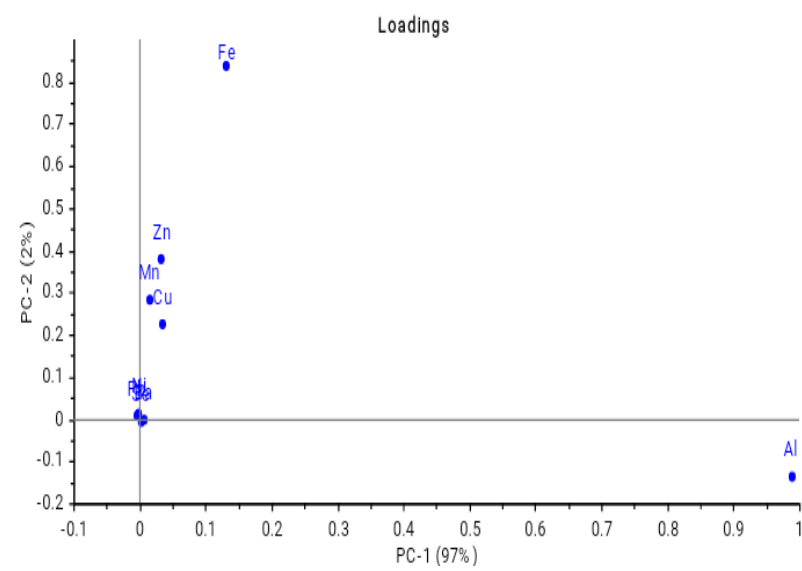

(b)

Fig. 2: Minor elements plot of the first principal components (PC1) versus the second principal component (PC2) for the triplicate of 10 dates -flesh samples: (a) scores plot and (b) loadings plot

G. Dates-Pit

\section{Major Elements}

The relationship between samples with major elements was observed by mapping the position of $\mathrm{Mg}, \mathrm{Na}, \mathrm{Ca}$ and $\mathrm{K}$ on PC1 of loadings plots with distribution of samples in scores plots as shown in Fig. 3, where PC1 and PC2 accounted $82 \%$ and $15 \%$ variance respectively in PCA model. The high content of those elements can be related to samples such as Ajwa to $\mathrm{Na}$; Medjool and Ajwa to $\mathrm{Ca}$; and Nourina to $\mathrm{Mg}$. Thus, it can be seen that the values $(\mathrm{mg} / \mathrm{kg}$ ) of $\mathrm{Mg}, \mathrm{Na}$ and $\mathrm{Ca}$ were high in Ajwa $(717,332,284)$, Medjool (614, 203, 273) and Nourina (629, 113, 208) respectively. While, $\mathrm{K}$ was observed high level in Khurdy $(1908 \mathrm{mg} / \mathrm{kg})$. 


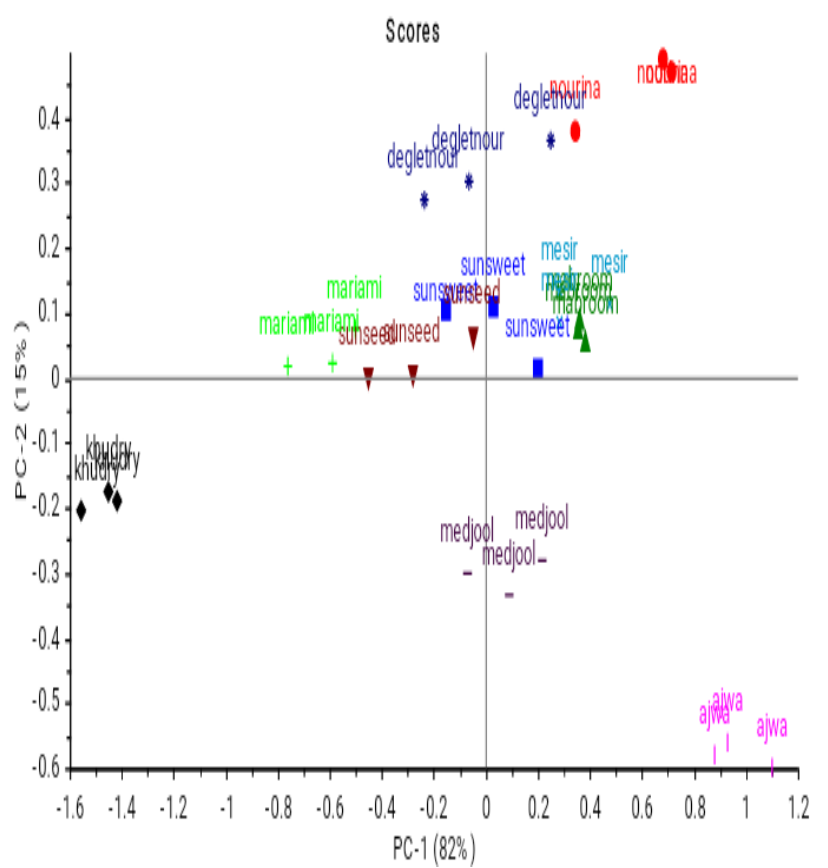

(a)

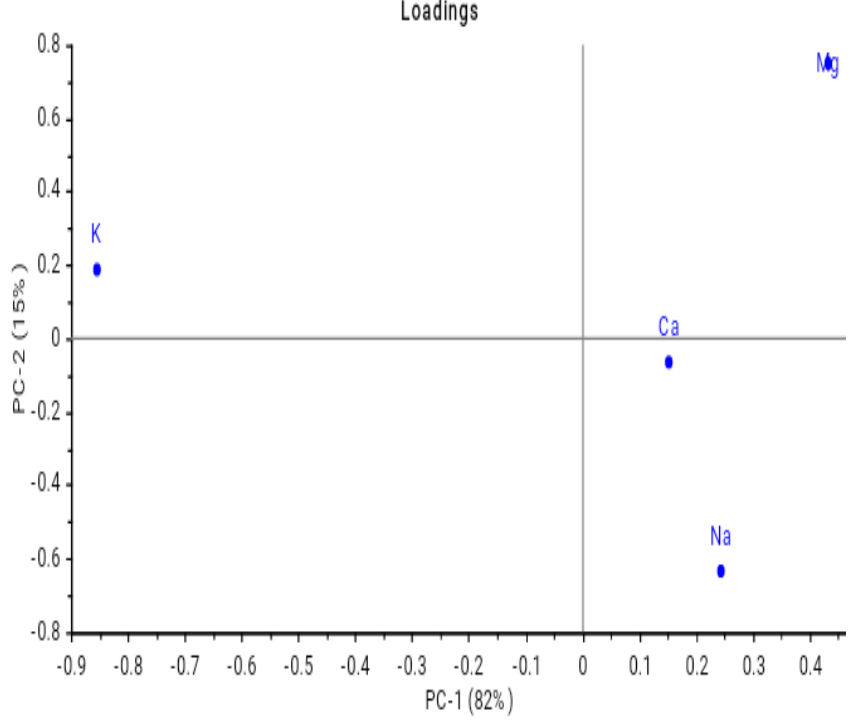

(b)

Fig. 3: Major elements plot of the first principal components (PC1) versus the second principal component (PC2) for the triplicate of 10 dates -pits samples: (a) scores plot and (b) loadings plot

\section{Minor Elements}

The relationship of samples and minor elements in datespit was observed in scores and x-loadings plots as shown in Fig. 4. Ajwa was found high in $\mathrm{Fe}(79.96 \mathrm{mg} / \mathrm{kg})$ and $\mathrm{Al}$ $(15.13 \mathrm{mg} / \mathrm{kg})$ whereas Medjool contained high levels of $\mathrm{Cu}$ (39.91 $\mathrm{mg} / \mathrm{kg}$ ) and $\mathrm{Zn}(36.33 \mathrm{mg} / \mathrm{kg})$. It was found that Khudry located close to Medjool as its $\mathrm{Cu}$ and $\mathrm{Zn}$ levels were $33.82 \mathrm{mg} / \mathrm{kg}$ and $31.13 \mathrm{mg} / \mathrm{kg}$ respectively, which are not much different with Medjool. Mariami, Mesir and Nourina were found closely located in the upper part of scores plots which is related with $\mathrm{Mn}$ in x-loadings plot. The content of $\mathrm{Mn}$ in respective samples was $9.57 \mathrm{mg} / \mathrm{kg}, 10.87$ $\mathrm{mg} / \mathrm{kg}$ and $9.66 \mathrm{mg} / \mathrm{kg}$. Other samples such as Mabroom, Negletnour, Sunsweet and Sunseed located in the middle of scores plot were found related with $\mathrm{Ni}, \mathrm{Pb}, \mathrm{Ba}$ and $\mathrm{Se}$ in $\mathrm{X}$ loadings plot where their values were closely similar.

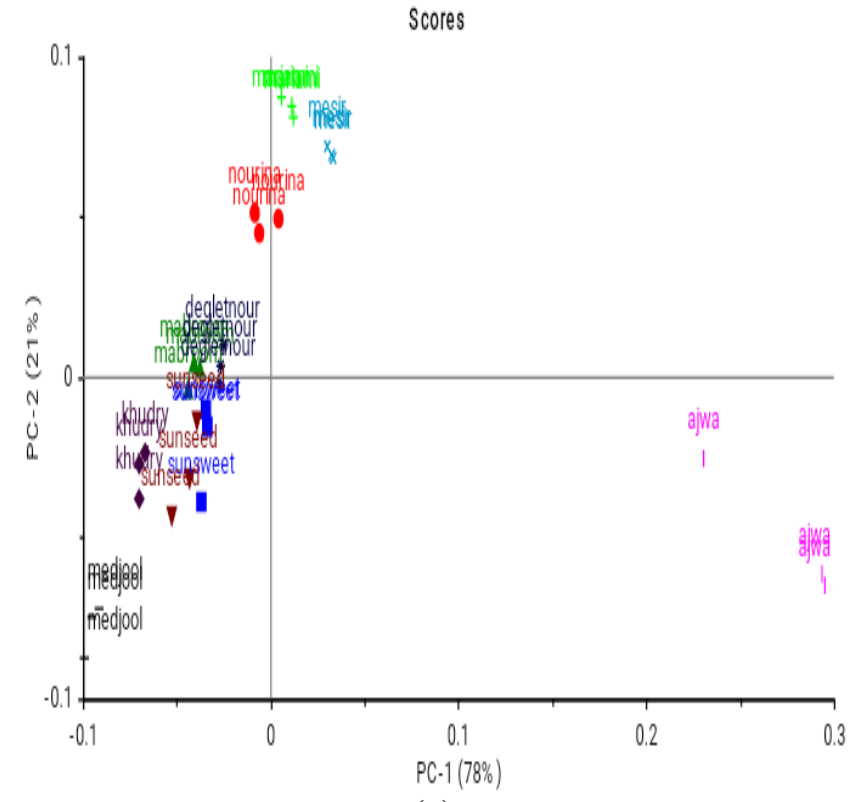

(a)

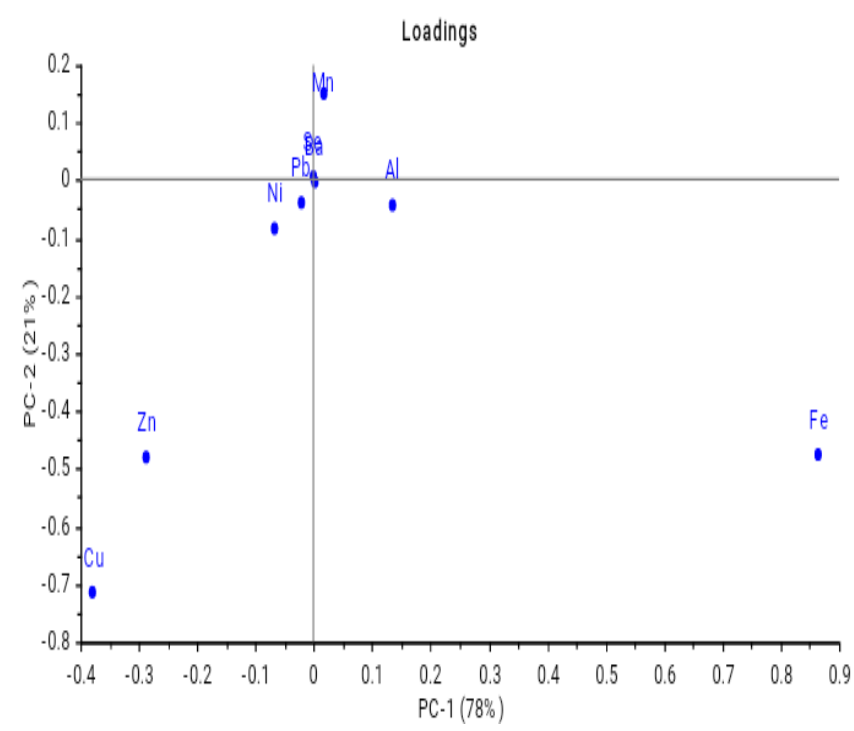

(b)

Fig. 4: Minor elements plot of the first principal components (PC1) versus the second principal component (PC2) for the triplicate of 10 dates -pits samples: (a) scores plot and (b) loadings plot

\section{CONCLUSION}

Hence, this study provides the elemental composition of dates fruit according to different varieties, purchased from Malaysia's supermarket. Both flesh and pits were determined for their minerals content and results revealed that $\mathrm{K}$ was the most abundant element $(5223 \mathrm{mg} / \mathrm{kg}$ for flesh and $1908 \mathrm{mg} / \mathrm{kg}$ for the pit). Based on the PCA plot, elements contained different varieties of dates sample was able to evaluate. The major elements content in dates are $\mathrm{K}$, $\mathrm{Mg}, \mathrm{Na}$ and $\mathrm{Ca}$ while other elements were observed in minor concentrations. Both Ajwa pits and flesh contained high concentration of $\mathrm{Ca}, \mathrm{Na}$ and $\mathrm{Mg}$. Ajwa, Medjool and Mesir dates-flesh proven to be different from other samples

Published By: 


\section{ASSESSMENT OF MINERALS IN PHOENIX DACTYLIFERA L. AS DETERMINED BY INDUCTIVELY COUPLED PLASMA OPTICAL EMISSION SPECTROMETRY USING ANOVA AND PCA}

in both major and minor elements. While for dates-pit, the differences were showed in Ajwa, Medjool and Khudry.

\section{ACKNOWLEDGMENT}

The authors would like to thank the Ministry of Higher Education, Malaysia for funding this research through Niche Research Grants Scheme (USIM/NRGS_P5/ISI/8405/52113).

\section{REFERENCES}

1. I. A. Ahmed, A. W. Ahmed, and R. K. Robinson, "Chemical composition of date varieties as influenced by the stage of ripening," Food Chemistry, 54(3), 1995, pp. 305-309.

2. F. Guido, S. E. Behija, I. Manel, Z. Nesrine, F. Ali, H. Mohamed, H. A. Noureddine, and A. Lotfi, "Chemical and aroma volatile compositions of date palm (Phoenix dactylifera L.) fruits at three maturation stages," Food Chemistry, 127(4), 2011, pp. 1744-1754.

3. W. Braide, S. U. Oranusi, and C. C. Otali, "Nutritional, antinutritional, minerals and vitamin compositions of fourteen brands of fruit juice sold in Onitsha main market," FS J Res Basic and App Sci., 1(3), 2012, pp. 46.

4. I. J. Salma, M. A. Sajib, M. Motalab, B. Mumtaz, S. Jahan, M. M. Hoque, and B. K. Saha, "Comparative evaluation of macro and micro-nutrient element and heavy metal contents of commercial fruit juices available in Bangladesh," American Journal of Food and Nutrition, 3(2), 2015, pp. 56-63.

5. F. A. Fazli, and N. A. Fazli, "Evaluation and determination of minerals content in fruits," International Journal of Plant, Animal and Environmental Sciences, 4(2), 2014, pp. 160-166.

6. D. Eruvbetine, "Canine nutrition and health," Seminar of Kensington Pharmaceuticals Nig. Ltd., 2003.

7. R. K. Murray, D. K. Granner, P. A. Mayes, and V. W. Rodwell, Harper's Biochemistry. New York: McGrawHill, 2000.

8. K. O. Soetan, C.O. Olaiya, and O. E. Oyewole, "The importance of mineral elements for humans, domestic animals and plants-A review," African Journal of Food Science, 4(5), 2010, pp. 200-222.

9. World Health Organization (WHO), The World Health Report 2001: Mental health: New understanding, new hope. Geneva: WHO, 2001.

10. M. S. Jamil, R. Nadeem, M. A. Hanif, M. A. Ali, and K. Akhtar, "Proximate composition and mineral profile of eight different unstudied date (Phoenix dactylifera L.) varieties from Pakistan," African Journal of Biotechnology, 9(22), 2010, pp. 3252-3259.

11. M. N. Al-Seeni, "Minerals content and antimicrobial efficacy of date extracts against some pathogenic bacteria," Life Science Journal, 19(2), 2012, pp. 504-508.

12. O. S. Agboola, and A. L. Adejumo, "Nutritional composition of the fruit of the Nigerian wild date palm, Phoenix dactylifera," World Journal of Dairy and Food Sciences, 8(2), 2013, pp. 196-200.

13. M. A. Sahari, M. Barzegar, and R. Radfar, "Effect of varieties on the composition of dates (Phoenix dactylifera L.) -note," Food Science and Technology International,

14. R. Bhat, K. Kiran, A. B. Arun, and A. A Karim, "Determination of mineral composition and heavy metal content of some nutraceutically valued plant products," Food Analytical Methods, 3(3), 2010, pp. 181-187.

15. H. Altundag, and M. Tuzen, "Comparison of dry, wet and microwave digestion methods for the multi element determination in some dried fruit samples by ICP-OES," 13(4), 2007, pp. 269-275.

Food and Chemical Toxicology, 49(11), 2011, pp. 2800 2807.

16. K. K. Taha, and F. M. Al Ghtani, "Determination of the elemental contents of date palm (Phoenix dactylifera L.) from Kharj Saudi Arabia," World Scientific News, 12, 2015, pp. 125-135.

17. G. Tyler, and S. J. Yvon, ICP-OES, ICP-MS and AAS techniques compared. ICP Optical Emission Spectroscopy Technical Note, New Jersey: Kyoto: Horiba Group, 1995.

18. G. Baskaran, N. A. Masdor, M. A. Syed, and M. Y Shukor, "An inhibitive enzyme assay to detect mercury and zinc using protease from Coriandrum sativum," Scientific World Journal, 2013, 2013, pp. 1-7.

19. E. A. Assirey, "Nutritional composition of fruit of 10 date palm (Phoenix dactylifera L.) cultivars grown in Saudi Arabia," Journal of Taibah University for Science, 9(1), 2015, pp. 75-79.

20. F. Y. Al-Juhaimi, S. H. Hamad, I. S. Al-Ahaideb, M. M. Al-Otaibi, K. Ghafoor, T. Abbasi, and S. A. Abbasi, "Biogas production through the anaerobic digestion of date palm tree wastes-process optimization," BioResources, 9(2), 2014, pp. 3323-3333.

21. F. A. Juhaimi, K. Ghafoor, and M. M. Özcan, "Physical and chemical properties, antioxidant activity, total phenol and mineral profile of seeds of seven different date fruit (Phoenix dactylifera L.) varieties," International Journal of Food Sciences and Nutrition, 63(1), 2012, pp. 84-89.

22. R. G. Brereton, Chemometrics: Data Analysis for the Laboratory and Chemical Plant. New Jersey: John Wiley and Sons, 2003.

23. A. Kolasani, H. Xu, and M. Millikan, "Evaluation of mineral content of Chinese medicinal herbs used to improve kidney function with chemometrics," Food Chemistry, 127(4), 2011, pp. 1465-1471.

24. S. Zhang, Y. Wei, S. Wei, H. Liu, and B. Guo, "Authentication of Zhongning wolfberry with geographical indication by mineral profile," International Journal of Food Science and Technology, 52(2), 2017, pp. 457-463.

25. I. Coelho, A. Rego, S. Gueifão, A. C. Nascimento, and I. Castanheira, "Application of chemometric methods for multi-elemental characterization of fruit juices and nectars analysed in the Portuguese Total Diet Studies pilot study," Journal of Chemometrics, 31(2), 2017, pp. $1-11$.

26. S. P. Kek, N. L. Chin, S. W. Tan, Y. A. Yusof, and L. S. Chua, "Classification of honey from its bee origin via chemical profiles and mineral content," Food Analytical Methods, 10(1), 2017, pp. 19-30.

27. A. G. Potortí, V. L. Turco, M. Saitta, G. D. Bua, A Tropea, G. Dugo, and G. D. Bella, "Chemometric analysis of minerals and trace elements in Sicilian wines from two different grape cultivars," Natural Product Research, 31(9), 2017, pp. 1000-1005.

28. C. G. Novaes, I. L. da Silva Romão, B. G. Santos, J. P. Ribeiro, M. A. Bezerra, and E. G. da Silva, "Screening of Passiflora L. mineral content using principal component analysis and Kohonen self-organizing maps," Food Chemistry, 233, 2017, pp. 507-513.

29. S. Parvin, D. Easmin, A. Sheikh, M. Biswas, S. C. Sharma, M. G. Jahan, M. A. Islam, M. S. Shovon, and N. Roy, "Nutritional analysis of date fruits (Phoenix dactylifera L.) in perspective of Bangladesh," American Journal of Life Sciences, 3(4), 2015, pp. 274-278. 
30. A. Uba, M. I. Abdullahi, A. J. Yusuf, Z. Y. Ibrahim, M. Lawal, I. Nasir, and F. T. Abdullahi, "Mineral profile, proximate and amino acid composition of three date's varieties (Phoenix dactylifera L.)," Derpharma, 7(5), 2015, pp. 3-48.

31. V. B. Vouk, and W. T. Piver, "Metallic elements in fossil fuel combustion products: Amounts and form of emissions and evaluation of carcinogenicity and mutagenicity," Environmental Health Perspectives, 47, 1983, pp. 201-225.

32. A. Celik, A. A. Kartal, A. Akdoğan, and Y. Kaska, "Determining the heavy metal pollution in Denizli (Turkey) by using Robinio pseudo-acacia L.," Environment International, 31(1), 2005, pp. 105-112.

33. S. S. Abdrabo, G. Grindlay, L. Gras, and J. Mora, "Multi-element analysis of Spanish date palm (Phoenix dactylifera L.) by inductively coupled plasma-based techniques. Discrimination using multivariate statistical analysis," Food Analytical Methods, 8(5), 2015, pp. 1268-1278.

34. P. Mariyam, and V. Mary, "Nutritional analysis (macronutrients, potassium and iron content) of four palm date varieties (phoenix dactylifera 1.) and study of consumption pattern among muslim and maharashtrian community," Journal of Food Processing and Beverages, 3(1), 2015, pp. 1-9.

35. B. Al-Gboori, and V. Krepl, "Importance of date palms as a source of nutrition," Agric. Trop. Subtrop., 43(4), 2010, pp. 348-351.

36. BfR, BfR Health Assessment No. 034: Aluminium in apple juice: No storing of fruit juice in aluminium tanks. Available:

http://www.bfr.bund.de/cm/349/aluminium_in_apple_jui ce_no_storing_of_fruit_juice_in_aluminium_tanks.pdf.

37. D. M. Trabzuni, S. E. Ahmed, and H. M. Abu-Tarboush, "Chemical composition, minerals and antioxidants of the heart of Date Palm from three Saudi cultivars," Food and Nutrition Sciences, 5(14), 2014, pp. 1379-1386.

38. H. A. Schroeder, I. H. Tipton, and A. P. Nason, "Trace metals in man: Strontium and barium," Journal of Chronic Diseases, 25(9), 1972, pp. 491-517.

39. World Health Organization (WHO), Cancer pain relief: With a guide to opioid availability. Geneva: WHO, 1996.

40. S. Al-Hooti, J. S. Sidhu, and H. Qabazard, "Physicochemical characteristics of five date fruit cultivars grown in the United Arab Emirates," Plant Foods for Human Nutrition, 50(2), 1997, pp. 101-113.

41. N. H. Jamil, I. Izzuddin, Z. Zainuddin, and M. H. Jumali, "Microstructural studies of nanocrystalline barium zirconium titanate (BZT) for piezoelectric applications," AIP Conference Proceedings, 1678(1), 2015, pp. 1-5.

42. R. M. Mohamed, A. S. Fageer, M. M. Eltayeb, and I. A. M. Ahmed, "Chemical composition, antioxidant capacity, and mineral extractability of S udanese date palm (P hoenix dactylifera L.) fruits," Food Science and Nutrition, 2(5), 2014, pp. 478-489.

43. Food and Nutrition Board, 2001.

44. W. Al-Shahib, and R. J. Marshall, "Fatty acid content of the seeds from 14 varieties of date palm Phoenix dactylifera L.," International Journal of Food Science and Technology, 38(6), 2003, pp. 709-712.

45. J. S. Hamada, I. B. Hashim, and F. A. Sharif, "Preliminary analysis and potential uses of date pits in foods," Food Chemistry, 76(2), 2002, pp. 135-137. 